\title{
Genetic polymorphism of N-acetyltransferase-2, glutathione S-transferase-M1, and cytochromes P450IIE1 and P450IID6 in the susceptibility to head and neck cancer
}

\author{
M V González, V Alvarez, M F Pello, M J Menéndez, C Suárez, E Coto
}

\begin{abstract}
Aims-To analyse the allele frequencies of DNA polymorphisms at the genes for cytochromes P450IIE1 and P450IID6, $\mathrm{N}$-acetyltransferase-2, and glutathione S-transferase-M1 in patients with head and neck squamous cell carcinoma, in an attempt to define genetic factors involved in the susceptibility to this cancer, which is strongly associated with tobacco consumption.

Methods-Determination of restriction fragment length polymorphism (RFLP) at cytochromes P450IIE1/P450IID6 and NAT2 genes, and the presence of homozygous deletion of the GSTM1 gene, in 200 controls and 75 head and neck cancer patients. Allelic frequencies between the two groups were compared using a $\chi^{2}$ test, and odds ratio with $95 \%$ confidence intervals were calculated.

Results-There was no evidence of an association between alleles of CYP2D6 and CYP2E1 and head and neck cancer in our population. Similarly, frequencies of individuals lacking the GSTM1 gene did not differ between controls and patients. However, individuals with the NAT2-SA phenotype were at higher risk of developing head and neck cancer. The frequencies of the most common SA genotype (homozygous for the NAT $2 \star 5$ allele) were higher in patients than in controls $(27 \% v 15 \%$, respectively). Slow acetylators homozygous for the NAT $2 \star 6$ allele, the second most common $S A$ allele, were also more common in patients than in controls $(11 \%$ $v 5 \%$, respectively).
\end{abstract}

Hospital Central de Asturias, 33006 Oviedo, Spain Laboratorio de Genética Molecular M V González V Alvarez

M J Menéndez E Coto

Servicio de ORL M F Pello

C Suárez

Correspondence to: Dr Eliecer Coto, Laboratorio de Genética Molecular, Hospital Central de Asturias, 33006 Oviedo, Spain.

Accepted for publication 13 January 1997 Conclusions-Slow NAT2 activity is a risk factor possibly leading to the development of head and neck cancer in response to tobacco carcinogens.

(F Clin Pathol 1998;51:294-298)

Keywords: head and neck squamous cell carcinoma; $\mathrm{N}$-acetyltransferase; glutathione S-transferase; cytochrome P450IIE1; cytochrome P450IID6

Squamous cell carcinoma of the head and neck is one of the commonest human neoplasms. Epidemiological studies have shown that this cancer type is associated with tobacco and alcohol consumption, and is commonest in regions where these products are most often consumed. Chemical compounds present in tobacco and alcohol undergo metabolic activa- tion by phase I enzymes, usually the cytochrome P450 enzymes (CYP). CYP catalyse reactions that result in the creation of functional groups on their substrates, thus generating the ultimate carcinogen. These carcinogens form DNA adducts, leading to genetic damage. Other enzymes - the phase II enzymes - act on these carcinogens, transforming them into inactivated compounds that are excreted. The action of phase II enzymes, such as glutathione S-transferases (GST) and N-acetyl transferases (NAT) generally contributes to detoxifying metabolism. In addition to their detoxifying activity, GSTs and NATs can also mediate activating steps for some carcinogen metabolites. ${ }^{1}$

Human cytochrome P450 is a superfamily of more than 100 different haem containing monomeric enzymes. Based on sequence homologies, human CYP are divided into 10 families. Variation in the enzymatic activity has been found for most of the CYP, thus determining interindividual differences in the metabolic capacity and cancer risk.

CYP2D6 oxidises debrisoquine, and two phenotypes have been established: poor metabolisers (PM) and extensive metabolisers (EM). PM individuals lack debrisoquine 4-hydroxylase activity, owing to a complete absence of an active form of the CYP2D6 enzyme. ${ }^{2}$ The most frequent PM allele in whites is a G to A transition at the junction of intron 3/exon 4 of the CYP2D6 gene. Several studies have described an association between the EM phenotype and the incidence of lung, bladder, and breast cancers. ${ }^{3-5}$ Recently, a higher risk of developing primary liver cancer among individuals homozygous for functional CYP2D6 genes has also been described. ${ }^{6}$

CYP2E1 catalyses oxidation and DNA adduct formation of several compounds found in cigarette smoke, such as $\mathrm{N}$-nitrosamines and benzene. CYP2E1 is also involved in the metabolism of ethanol and acetone. Genetic and racial variations in the CYP2E1 gene have been described. ${ }^{7}$ One of these variants, identified by a PstI-RFLP at the 5'-promoter region, correlated with the transcriptional activity of the gene, defining the "low expression" (LE) and "high expression" (HE) CYP2E1 alleles. The increased expression of CYP2E1 could be a factor contributing to susceptibility to lung cancer. $^{8}$

$\mathrm{N}$-acetyltransferases have been linked to the detoxification of several dietary and occupational arylamine carcinogens, primarily functioning as phase II conjugation enzymes. There 
are two functional NAT enzymes, NAT1 and NAT2. ${ }^{9}$ NAT2 is polymorphic, metabolises aromatic amines, and is involved in the phase II metabolism of several compounds such as caffeine. These compounds have been used in several studies to relate acetylation capacity to disease susceptibility. Between $40 \%$ and $70 \%$ of white people have the "slow acetylator" (SA) phenotype, and so are less efficient than "rapid acetylators" (RA) at metabolising numerous chemical carcinogens. Several mutations in the NAT2 gene affect the activity of the protein, and are responsible for the SA phenotype. These mutant forms can be determined by polymerase chain reaction (PCR)-restriction fragment length polymorphism (RFLP) analysis, and amino acid changes significantly reduce the maximum velocity of $\mathrm{N}$-acetyltransferase in these mutants compared with the NAT2 "wild type" allele. ${ }^{10-13}$ NAT2 is expressed in a wide range of human tissues, and the association between NAT2 activity and cancer susceptibilities has been described. Thus RA individuals are at a higher risk of developing colorectal cancer, whereas SA individuals are at a higher risk from bladder and breast cancer. ${ }^{14-16}$ The contradiction represented by the apparent predisposition of fast acetylators to colon cancer could be explained by the fact that, in addition to N-acetylation, NATs can also mediate activating steps for some carcinogen metabolites, such as $\mathrm{N}, \mathrm{O}$-transacetylation and O-acetylation. ${ }^{17}{ }^{18} \mathrm{An}$ association between the slow acetylator phenotype and laryngeal cancer has also been described. ${ }^{19}$

The glutathione S-transferases consist of a superfamily of enzymes that catalyse the reduction by glutathione of several electrophilic substrates, facilitating the excretion of these compounds. These compounds are often products of CYP mediated metabolism. The GST-mu class (GSTM) is the best known GST family. One of the GSTM genes, GSTM1, is homozygously deleted in up to $50 \%$ of white people. ${ }^{20}$ Several studies have described a higher risk of developing lung cancer among smokers with the null GSTM1 genotype. ${ }^{21} 22$

In this study, we investigated CYP2D6, CYP2E1, NAT2, and GSTM1 genotypes in healthy Spanish individuals and in patients with squamous cell carcinoma of the head and neck. We analysed the association between genetic polymorphisms and the development of this cancer type.

\section{Methods}

SUBJECTS

Seventy five patients affected by head and neck cancer (35 pharynx, 35 larynx, and five floor of the mouth) and 200 unselected controls from the same white population (Asturias, northern Spain) were analysed for CYP2D6, CYP2E1, NAT2, and GSTM1 genotypes. All patients were treated at the ORL Unit of the Hospital Central de Asturias in the 1993-1994 period. All patients were male, and smoking habits, alcohol consumption, and occupational history were obtained. Healthy blood donors (150 male and 50 female)-recruited through the molecular genetics laboratory of the Hospital
Central de Asturias-were used as controls. These controls were selected for a case-control study of risk factors for head and neck cancer. ${ }^{23}$ Informed consent was obtained from patients and controls.

RFLP ANALYSIS OF THE CYP GENES

DNA was isolated from leucocytes in $10 \mathrm{ml}$ of peripheral blood. PCR was used to amplify fragments of the CYP2D6, CYP2E1, NAT2, and GSTM1 genes. Reactions consisted of 0.2 $\mu \mathrm{g}$ of genomic DNA, $30 \mathrm{pmol}$ each primer, 0.2 $\mathrm{mM}$ each dNTP, $1.5 \mathrm{mM} \mathrm{MgCl}_{2}, 1 \times$ Taq buffer, and $1 \mathrm{U}$ of Taq DNA-polymerase (Promega) to a final volume of $30 \mu \mathrm{l}$. After 30 cycles of $98^{\circ} \mathrm{C}$ for 30 seconds, annealing temperature for one minute, and $72^{\circ} \mathrm{C}$ for one minute, followed by a final extension of $72^{\circ} \mathrm{C}$ for five minutes, $10 \mu \mathrm{l}$ of PCR were digested with $10 \mathrm{U}$ of the appropriate restriction enzyme and electrophoresed on a $2 \%$ agarose gel.

A $335 \mathrm{bp}$ fragment containing the intron 3/exon 4 junction of the CYP2D6 gene was amplified, and the $G$ to $A$ transition at this intron/exon junction responsible for the most common CYP2D6-PM allele was analysed as described. ${ }^{23}$ Briefly, genomic DNA was amplified with primers GCCTTCGCCAACCACTCCG and AAATCCTGCTCTTCCGAGGC (annealing temperature $60^{\circ} \mathrm{C}$ ) and digested with BstNI. The EM allele gives two fragments of 230 and 105, while the PM allele is not digested.

PCR was also used to analyse CYP2E1 polymorphism. ${ }^{78}$ A $410 \mathrm{bp}$ fragment from the transcription regulatory region of CYP2E1 was amplified with primers CCAGTCGAGTCTACATTGTCA and TTCATTCTGTCTTCTAACTGG (annealing at $60^{\circ} \mathrm{C}$ ), and digested with PstI. A site for this enzyme is present in the c2 allele and absent in the $\mathrm{c} 1$ allele (c1 and c2 are high expression and low expression alleles, respectively).

RFLP ANALYSIS OF THE NAT2 GENE

A C to T change at position 481 of the NAT2 gene that eliminates a target sequence for KpnI is the most common mutation associated with the slow acetylator phenotype. This allele is designated NAT2 ${ }^{\star} 5$ and was identified by KpnI digestion of a 290 bp PCR fragment obtained with primers TGTCGATGCTGGGTCTGGAA and ATGAAGATGTTGGAGACGT (annealing temperature $62^{\circ} \mathrm{C}$ ). These PCR primers were derived from the NAT2 sequence (EMBL accession number $\mathrm{X} 14672)$. After digestion with $\mathrm{KpnI}$, the 481-T (NAT2 ${ }^{\star} 5$ ) and $481-\mathrm{C}$ alleles are visualised as fragments of $290 \mathrm{bp}$ and $170+120 \mathrm{bp}$, respectively. A G to A change at nucleotide 590 is the second most common SA allele, designated NAT ${ }^{\star} 6$. This mutation destroys a TaqI site. For the analysis of this mutation genomic DNA from patients and controls was amplified with the NAT2 primers described above and digested with TaqI. The 590-A (NAT2 ${ }^{\star} 6$ ) and $590-\mathrm{G}$ alleles are visualised as fragments of 290 and $230+60 \mathrm{bp}$, respectively. 
PCR ANALYSIS OF THE GSTM1 GENE

PCR with primers CTGCCCTACTTGATTGATGGG and CTGGATTGTAGCAGATCATGC (annealing at $55^{\circ} \mathrm{C}$ ) was used to determine the presence or absence (homozygous deletion) of the GSTM1 gene, according to previously described procedures. ${ }^{20}$

\section{STATISTICAL METHODS}

The $\chi^{2}$ test was used to assess the distribution of allele frequencies between the groups. Odds ratios (OR) and their 95\% confidence intervals (CI) were calculated using the BMPD New Systems for Windows, version 1.0 (BMDP, Cork, Ireland).

\section{Results}

Table 1 summarises the age, smoking, and alcohol data on cases and controls. Of the controls, $30 \%$ and $10 \%$ were "occasionally" smokers and drinkers, respectively. All of the 75 patients were male and smokers, $80 \%$ of them also "heavy" drinkers, with an average age of 58.7 years (range 35 to 81 years). The average number of cigarettes consumed was 59 packs/ day $\times$ years consumed (range 27 to 180 ), with the highest consumption in an 81 year old patient. The average alcohol consumption among "heavy" drinkers was 87 litres of wine/ day $\times$ years consumed (range 70 to 122 ), with the highest consumption in a 65 years old patient

Table 2 summarises the data on the genotype frequencies for the RFLP at the CYP2D6, CYP2E1, and NAT2 genes, as well as for homozygous deletion of the GSTM1 gene. No difference was found in the genotype frequencies among controls with respect to gender (150 males $v 50$ females). Genotype frequencies (CYP2D6, CYP2E1, and NAT2) were in the Hardy-Weimberg equilibrium in the control population. Comparison of control and head and neck cancer patients showed no significant differences for the CYP2D6, CYP2E1, and GSTM1 polymorphisms.

The CYP2E1 LE-allele, recognised by the absence of a PstI site on the amplified sequence, had a frequency of 0.957 and 0.940 in controls and patients, respectively. The seven patients who carried the HE allele were found among both the "heavy" drinkers (LE/HE, four cases) and among the "occa-

Table 1 Summary of smoking and alcohol history in the head and neck squamous cell carcinoma patients and the control group

\begin{tabular}{llc}
\hline & $\begin{array}{l}\text { Cancer patients } \\
(n=75)\end{array}$ & $\begin{array}{l}\text { Controls } \\
(n=200)\end{array}$ \\
\hline Age, mean (range) years & $58.7(35$ to 81$)$ & 45 (25 to 75) \\
Smoking history & 0 & 135 \\
$\quad$ Non-smokers & 40 & 65 \\
Light smokers (a) & 35 & 0 \\
$\quad$ Heavy smokers (b) & & \\
Alcohol consumption & 15 & 200 \\
$\quad$ Occasional & 60 & 0 \\
Heavy (c) &
\end{tabular}

(a) Subjects who smoked below the average cigarette consumption of the patients (59 packs/day $\times$ years consumed). (b) Subjects who smoked above the average cigarette consumption.

(c) Subjects who drank above the lower limit in "heavy" drinker patients (70 litres of wine/day $\times$ years).
Table 2 DNA polymorphism genotypes in 200 controls and 75 HNSCC patients (percentages in parentheses)

\begin{tabular}{lccl}
\hline & Patients & Controls & $p$ Value \\
\hline CYP2E1 (a) & & & \\
LE/LE & $68(90)$ & $179(90)$ & NS \\
LE/HE & $6(8)$ & $21(10)$ & \\
HE/HE & $1(2)$ & 0 & \\
CYP2D6 (b) & & & \\
PM/PM & $3(4)$ & $7(3)$ & NS \\
PM/PM- & $11(14)$ & $40(21)$ & \\
PM-/PM- & $61(82)$ & $153(76)$ & \\
GSTM1 (c) & & & \\
- & $44(58)$ & $103(54)$ & NS \\
+ & $31(42)$ & $97(46)$ & \\
NAT $\star 5$ (d) & & & \\
$\star 5 / * 5$ & $20(27)$ & $30(16)$ & $<0.04$ \\
$\star 5 /$ wt & $35(46)$ & $105(50)$ & \\
wt/wt & $20(27)$ & $65(34)$ & \\
NAT*6 (e) & & & \\
$\star 6 / \star 6$ & $8(11)$ & $7(5)$ & $<0.04$ \\
$\star 6 /$ wt & $32(42)$ & $89(44)$ & \\
wt/wt & $35(47)$ & $105(51)$ & \\
NAT2 (f) & & & \\
SA & $28(37)$ & $37(21)$ & $<0.002$ \\
RA & $47(63)$ & $163(79)$ & \\
\hline
\end{tabular}

(a) HE, high expression allele, presence of a PstI site; LE, low expression allele, absence of a PstI site.

(b) PM, poor metaboliser allele, absence of a BstNI site; PM-, wild type allele, presence of a BstNI site.

(c) GSTM1-, non-amplification of the GSTM1 gene; GSTM1+, amplification of the GSTM1 sequence.

(d) NAT2 ${ }^{\star}$, slow acetylator allele, absence of a KpnI site; wt, wild type allele, presence of a KpnI site.

(e) NAT $2^{\star} 6$, slow acetylator allele, absence of a TaqI site; wt, wild type allele, presence of a taqI site.

(f) NAT2, SA are defined as those individuals homozygous for the NAT $2{ }^{\star} 5$ and/or NAT $2{ }^{\star} 6$ alleles.

sional" drinkers (LE/HE, one case; HE/HE, one case). For the CYP2D6 gene the frequencies of the PM allele (absence of a BstNI site) were 0.136 and 0.110 in controls and patients, respectively. Forty six per cent of the controls $v$ $42 \%$ of the patients amplified the GSTM1 sequence (fig 1 ).

The NAT $2{ }^{\star} 5$ allele was present at frequencies of 0.42 and 0.50 in the control and patient populations, respectively. The NAT $2{ }^{\star} 6$ frequencies were 0.24 and 0.30 in controls and patients, respectively. Table 2 shows genotypic frequencies for the NAT2 polymorphisms. Individuals homozygous for the NAT $2^{\star} 5$ and NAT $2 \star 6$ alleles occurred more often in patients than in controls. Thus $15 \%$ of controls $v 27 \%$ of patients showed the NAT2 ${ }^{\star} 5 / \mathrm{NAT} 2{ }^{\star} 5$ genotype, and $5 \%$ of controls $v 11 \%$ of patients showed the NAT2 ${ }^{\star} 6 / \mathrm{NAT} 2{ }^{\star} 6$ genotype. In both cases, genotypic frequencies between controls and patients were significantly different (NAT2 $\left.{ }^{*} 5, \mathrm{p}<0.039 ; \mathrm{NAT}^{\star}{ }^{*} 6, \mathrm{p}<0.042\right)$. Odds ratios and their $95 \%$ confidence intervals between the patient and the control groups were $0.485(0.25$ to 0.93$)$ and $0.304(0.10$ to $0.89)$ for the NAT ${ }^{\star} 5 / \mathrm{NAT}^{\star}{ }^{\star} 5$ and NAT ${ }^{\star}{ }^{6} 6 /$ NAT $2 \star 6$ genotypes, respectively. Genotypic frequencies between the patients with laryngeal (35 cases) and pharyngeal tumours (35 cases) did not differ significantly (data not shown).

Previous studies have shown that NAT2 ${ }^{\star} 5$ / $\mathrm{NAT}^{\star}{ }^{\star} 5$ and $\mathrm{NAT}^{\star}{ }^{\star} 6 / \mathrm{NAT}^{\star}{ }^{\star} 6$ individuals have a significantly reduced NAT2 activity. ${ }^{10-12}$ Distribution of slow acetylators, defined as those homozygous for at least one of the NAT2 SA alleles, was also compared between patients and controls. SA frequencies were $21 \%$ (37/ $200)$ in controls and $37 \%(28 / 75)$ in patients, 


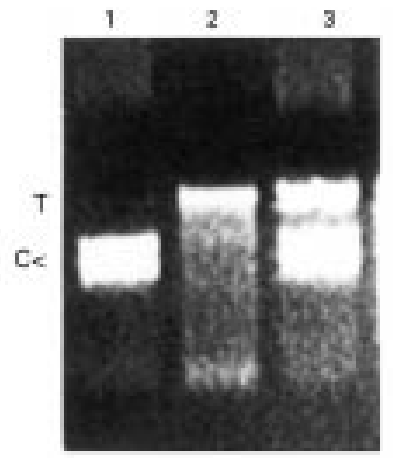

A

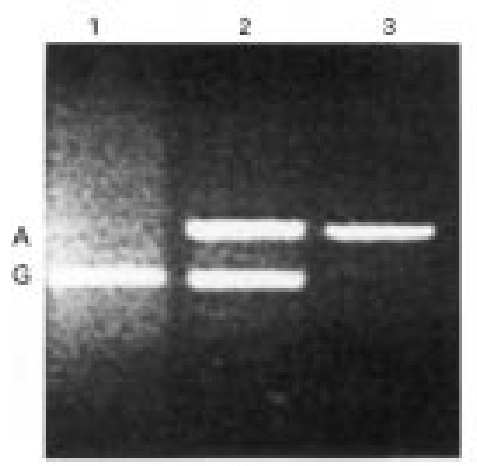

C

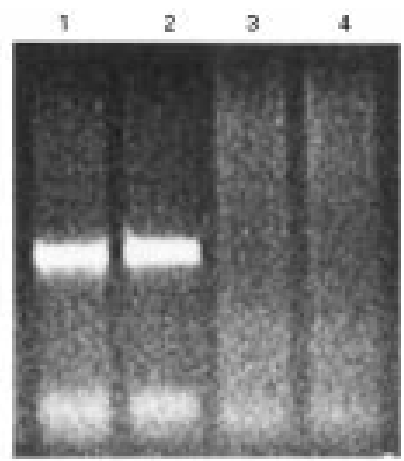

8

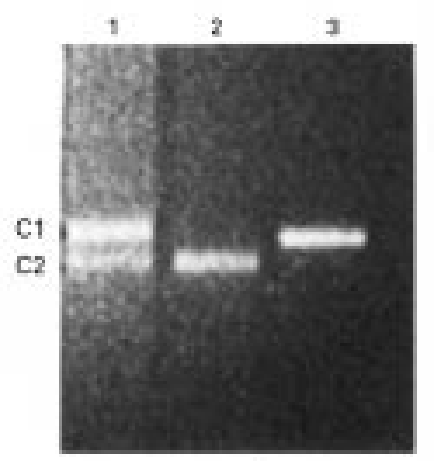

D

Figure 1 Homozygous and heterozygous individuals for the restriction fragment length polymorphism at the NAT2-KpnI site (481C to T) (A);CYP2D6-BstN1 site (intron3/exon 4 junction, $G$ to $T$ ) (C); and CYP2E1-PstI site (D). (B) Polymerase chain reaction (PCR) of the GSTM1 gene on DNA from lymphocytes of two GSTM1+ patients (lanes 1 and 2) and two GSTM1-null patients (lanes 3 and 4, homozygous deletion of the GSTM1 gene).

Table 3 Distribution of slow and rapid acetylators among patients who smoked below or above the median tobacco consumption. Slow acetylators were defined as those homozygous for the $N A T 2{ }^{*}$ and/or the $N A T 2{ }^{\star} 6$ alleles (percentages in parentheses)

\begin{tabular}{lll}
\hline & Below $(n=37)$ & Above $(n=38)$ \\
\hline SA & $18(48)$ & $10(26)$ \\
RA & $19(52)$ & $28(74)$ \\
\hline
\end{tabular}

$\mathrm{p}=0.078$, odds ratio $=0.377,95 \%$ confidence interval $=0.23$ to 1.01 (low smoking, rapid acetylators as the reference group)

respectively $(\mathrm{p}=0.002 ; \mathrm{OR}=0.38,95 \% \mathrm{CI}=$ $0.21,0.70$ ) (table 2).

We also analysed the genotype frequencies in patients with below or above the median tobacco or alcohol consumption. Frequencies of slow acetylators (individuals homozygous for the NAT2*5 and/or the NAT2* 6 allele) were 0.48 (18 of 37) and 0.26 (10 of 38) among patients below and above the median tobacco consumption, respectively (table 3). The difference between the two groups was not significant ( $p=0.078$ ). No significant differences between the two groups of patients were found for the GSTM1, CYP2E1, or CYP2D6 genotypes. The distribution of the CYP2E1 genotypes did not differ between patients below and above the median alcohol consumption (data not shown).

\section{Discussion}

The results of our work suggest the absence of an association of CYP2D6, CYP2E1, and

GSTM1 genotypes with head and neck cancer risk. Several studies have found a correlation between the CYP2D6-PM genotype and susceptibility to lung cancer. ${ }^{35}$ Ethnic differences between lung cancer susceptibility and CYP2E1 polymorphisms have been described. ${ }^{7}$ Owing to the low frequency of the CYP2E1-HE and CYP2D6-PM alleles the statistical power of our study, based on 75 patients and 200 controls, is very limited. We found that GSTM1 was homozygously deleted in $54 \%$ of controls and in $58 \%$ of the head and neck cancer patients. Previous studies have found marked racial differences for the null GSTM1 genotype association to lung cancer. ${ }^{2122}$

$\mathrm{N}$-acetyltransferases are important detoxifying phase 2 enzymes with an ubiquitous expression profile, suggesting a protective role in all the tissues exposed to carcinogens. Slow acetylator individuals would be at higher risk of developing head and neck cancer as a result of a reduced capacity to detoxify the chemical carcinogens generated by CYP and other enzymes. In our study we analysed the two most frequent NAT2 RFLP associated with the slow acetylator phenotype. The $\mathrm{C}$ to $\mathrm{T}$ change at nucleotide position 481 that determines the loss of a KpnI site is a silent mutation. However, approximately $80 \%$ of the chromosomes with this NAT2 mutation also have T to $\mathrm{C}$ and $\mathrm{A}$ to $\mathrm{G}$ changes at positions 341 and 803, respectively. ${ }^{13}$ These are not silent mutations (Ile-341 to Thr and Lys-803 to Arg) and the amino acid changes would be responsible for the slow acetylator phenotype. ${ }^{10-13}$ The second most frequent NAT2 slow acetylator allele consists in a G to A change at nucleotide 590. The amino acid $197:$ Arg $\rightarrow$ Glu change introduced by this mutation reduces the NAT2 activity to $24 \%$ of the NAT2 wild-type activity. ${ }^{12}$ Evidence for the involvement of NAT2 in the risk of developing head and neck cancer as a result of tobacco consumption is suggested by the fact that individuals homozygous for the slow acetylator alleles were significantly more frequent in the head and neck cancer patients than in the control population. In addition, we found an increased frequency of the NAT2 slow acetylator genotypes among patients who smoked below the median tobacco consumption compared with patients who smoked above the median. However, the differences were not statistically significant, probably owing to the limited number of patients analysed (table 3). NAT2 has been implicated in the development of bladder cancer, with the frequency of slow acetylators increasing from $44 \%$ in controls to $71 \%$ in bladder cancer patients exposed to occupational carcinogens. ${ }^{14}$ More recently, a higher frequency of the slow acetylator genotypes was found among postmenopausal woman who developed breast cancer. ${ }^{16}$ Contrary what happens in bladder and breast cancer, the NAT2 fast acetylator genotype has been found with a higher frequency among lung cancer patients. ${ }^{24}$

Head and neck cancer is a multifactorial disorder, and predisposition to the development of such disorders is likely to be a result of several genetic polymorphisms. ${ }^{25}$ Several tumour sup- 
pressor genes and oncogenes are involved in the development of head and neck cancer. ${ }^{26}$ Exposure to tobacco carcinogens contributes to the mutation of these genes. The reduced detoxifying capacity of "slow acetylators" would lead to a higher risk of mutations occurring at oncogenes and tumour suppressor genes, and as a consequence to a higher risk of developing head and neck cancer in the slow acetylator smokers. The metabolic pathways involved in the protective effect of acetylation have not been elucidated. It is also possible that this NAT protective activity is actually mediated by NAT1, a gene closely linked to NAT2 on chromosome $8 .^{28}$ If this is the case, the observed NAT2 association with cancer would be a result of genetic linkage disequilibrium. Because of the importance of NAT2 in the risk of developing head and neck cancer, further studies on other populations would be of special interest.

This work was supported by grants FISS 96/1418 and 97/1062 to EC. MVG is the recipient of a fellowship of the FICYT.

1 Smith CAD, Smith G, Wolf CR. Genetic polymorphisms in xenobiotic metabolism. Eur f Cancer 1994;30A:1921-35.

Smith CAD, Gough AC, Leigh PN, et al. Debrisoquine hydroxylase gene polymorphism and susceptibility to Parkinson's disease. Lancet 1992;339:1375-7.

3 Wolf CR, Smith CAD, Bishop T, et al. CYP2D6 genotyping and the association with lung cancer susceptibility. Pharmacogenetics 1994;4:104-6.

4 Caporaso NG, Hayes RB, Dosmeci M, et al. Lung cancer risk, occupational exposure and the debrosoquine metabolic phenotype. Cancer Res 1989;49:3675-9.

5 Caporaso NG, Tucker MA, Hoover RN, et al. Lung cancer and the debrisoquine metabolic phenotype. $\mathcal{f}$ Natl Cancer and the debrisoquine

6 Agúndez JAG, Ledesma MC, Benítez J, et al. CYP2D6 genes and risk of liver cancer. Lancet 1995;345:830-1.

7 Kato S, Shields PG, Caporaso NE, et al. Cytochrome P450IIE1 genetic polymorphisms, racial variation, and P450IIE1 genetic polymorphisms, racial var
lung cancer risk. Cancer Res 1992;52:6712-15.

8 Hirvonen A, Husgatvel-Pursiainen K, Anttila S, et al. Human CYP2E1 gene and lung cancer. DraI and Rsa restriction fragment length polymorphism in a Finnish study population. Carcinogenesis 1993;14:85-8.

9 Vineis D, Bartsch H, Caporaso N, et al. Genetically based $\mathrm{N}$-acetyltransferase polymorphism and low-level environmental exposure to carcinogens. Nature 1994;369:154-6.

10 Blum M, Demierre A, Grant DM, et al. Molecular mechanism of slow acetylation of drugs and carcinogens in humans. Proc Natl Acad Sci USA 1991;88:5237-41.
11 Hein DW, Doll MA, Rustan TD, et al. Metabolic activation of N-hydroxyarylamines and N-hydroxyarylamides by 16 recombinant human NAT2 allozymes: effects of 7 specific NAT2 nucleic acid substitutions. Cancer Res 1995;55: 3531-6.

12 Hein DW, Ferguson RJ, Doll MA, et al. Molecular genetics of human polymorphic $\mathrm{N}$-acetyltranferase: enzymatic analysis of 15 recombinant wild-type, mutant, and chimeric NAT2 allozymes. Hum Mol Genet 1994;3:729-34.

13 Cascorbi I, Drakoulis N, Brockmöller J, et al. Arylamine $\mathrm{N}$-acetyltransferase (NAT2) mutations and their allelic linkage in unrelated Caucasian individuals: correlation with phenotypic activity. Am f Hum Genet 1995;57:581-92.

14 Risch A, Wallace DMA, Bathers S, et al. Slow N-acetylation genotype is a susceptibility factor in occupational and smoking related bladder cancer. Hum Mol Genet 1995;4: 231-6.

15 Ilett KF, David BM, Detchon P. Acetylation phenotype in colorectal cancer. Cancer Res 1987;47:1466-9.

16 Ambrosone CB, Freudenheim JL, Graham S, Marshall JR, et al. Cigarette smoking, $\mathrm{N}$-acetyltransferase 2 genetic polymorphisms, and breast cancer. $7 A M A 1996 ; 276: 1494$ 501.

17 Hein DW. Acetylator genotype and arylamine-induced carcinogenesis. Biochim Biophys Acta 1988;948:37-66.

18 Hayes JD, Wolf CR. Molecular mechanisms of drug resistance. Biochem f 1990;272:281-95.

19 Drozdz M, Gierek T, Jendryczko A. N-acetyltransferase phenotype of patients with cancer of the larynx. Neoplasia 1987;34:481-4.

20 Comstock KE, Sanderson BJS, Claflin G, et al. GSTM1 gene deletion determined by polymerase chain reaction. Nucleic Acids Res 1990;18:3670.

21 Heckbert SR, Weiss NS, Horning SK, et al. Glutathione S-transferase and epoxide hydrolase activity in human leucocytes in relation to risk of lung cancer and other smoking related cancers. 7 Natl Cancer Inst 1992;84:414-22.

22 Seidegard J, Pero RW, Miller DW, et al. A glutathione transferase in human leukocytes as a marker for the susceptibility to lung cancer. Carcinogenesis 1986;7:751-3.

23 Norell SE. Workbook of epidemiology. New York: Oxford University Press, 1995.

24 Cascorbi I, Brockmöller J, Mrozikiewicz PM, et al. Homozygous rapid arylamine $\mathrm{N}$-acetyltransferase (NAT2) genotype as a susceptibility factor for lung cancer. Cancer Res 1996;56:3961-6.

25 Gonzalez FJ. Genetic polymorphism and cancer susceptibility: fourteenth Sapporo Cancer Seminar. Cancer Res 1995;55:710-15.

26 González MV, Pello MF, Suárez C, et al. Loss of heterozygosity and mutation analysis of the p16 (9p21) and p53 (17p13) genes in squamous cell carcinoma of the head and neck. Clin Cancer Res 1995;1:1043-9.

27 González MV, Pello MF, Suárez C, et al. Deletion and methylation of the tumor suppressor gene p16/CDKN2 in primary head and neck squamous cell carcinoma. $\mathcal{F}$ Clin Pathol 1997;50:509-12.

28 Hickman D, Risch A, Buckle V, et al. Chromosomal localization of human genes for arylamine $\mathrm{N}$-acetyltransferase. Biochem f 1994;297:441-5. 